\title{
Completion and decomposition of hypergraphs into dominating sets of graphs
}

\author{
Jaume Martí-Farré a,1,3, Mercè Mora b,2,4 and \\ José Luis Ruiz b,5 \\ a Departament de Matemàtica Aplicada IV \\ Universitat Politècnica de Catalunya \\ Barcelona, Spain \\ b Departament de Matemàtica Aplicada II \\ Universitat Politècnica de Catalunya \\ Barcelona, Spain
}

\begin{abstract}
The collection of the vertex dominating sets of a graph defines a hypergraph on the set of vertices of the graph. However, there are hypergraphs $H$ that are not the collection of the vertex dominating sets of any graph. This paper deals with the question of completing these hypergraphs $H$ to the vertex dominating sets of some graphs $G$. We demonstrate that such graphs $G$ exist and, in addition, we prove that these graphs define a poset whose minimal elements provide a decomposition of $H$. Moreover, we show that the hypergraph $H$ is uniquely determined by the minimal elements of this poset. The computation of such minimal elements is also discussed in some cases.
\end{abstract}

Keywords: Graph, hypergraph, dominating set. 


\section{Introduction}

A vertex dominating set of a graph $G$ is a set of vertices $D$ such that every vertex of $G$ is either in $D$ or adjacent to some vertex of $D$. Domination in graphs is a widely researched branch of graph theory, both from a theoretical and algorithmic point of view (see $[6,7,8]$ ). In part, it is due to its applications to several fields where graphs are used to model the relationships between a finite number of objects.

A great many theoretical issues, properties and algorithms have been studied regarding domination in graphs. The wide variety of papers that deal with parameters is remarkable; they range from the standard domination number of a graph to other domination-related parameters (see [8]). There are also many algorithmic papers addressing classes of graphs for which these parameters can be computed in polynomial time, as well as papers providing combinatorial bounds and algorithms for enumerating all the vertex dominating sets of certain graphs (see $[2,3,4,5,9]$ ).

This paper fits within this context and stems from a theoretical question related with domination in graphs whose solution will lead us to consider a new domination-related parameter, as well as to several issues regarding its computation. The starting point of this work is a question concerning the design of networks on a finite set of nodes $\Omega$ whose dominating sets satisfy specific properties. Thus, in this paper we focus our attention on the collection $\mathcal{D}(G)$ of all the inclusionwise minimal vertex dominating sets of a graph $G$. Specifically, we are looking for graphs $G$ whose collection of vertex dominating sets $\mathcal{D}(G)$ is equal or close to a given collection $\left\{A_{1}, \ldots, A_{r}\right\}$ of subsets of nodes $A_{i} \subseteq \Omega$.

Hypergraphs become the natural framework of this problem. A hypergraph $H$ on a finite set $\Omega$ is a collection of subsets of $\Omega$ none of which is a proper subset of another (see [1]). The domination hypergraph of a graph $G$ is the collection $\mathcal{D}(G)$ of all the inclusion-minimal vertex dominating sets of a graph $G$. A hypergraph $H$ is said to be a domination hypergraph if $H$ is the domination hypergraph of a graph; that is, if $H=\mathcal{D}(G)$ for some graph $G$.

\footnotetext{
1 Partially supported by projects MINECO MTM2011-28800-C02-01 and Gen. Cat. DGR 2014SGR1147.

2 Partially supported by projects MINECO MTM2012-30951/FEDER and Gen. Cat. DGR 2014SGR46.

3 Email: jaume.marti@ma4.upc.edu

4 Email: merce.mora@upc.edu

5 Email: jose.luis.ruiz@upc.edu
} 
In general, a hypergraph $H$ is far from being the domination hypergraph of a graph. Therefore, a natural question that arises at this point is to seek domination completions of a hypergraph $H$, that is, to look for graphs $G$ whose associated domination hypergraphs $\mathcal{D}(G)$ are close to the hypergraph $H$. Specifically, the goal is to prove that these domination completions exist, and moreover, by taking into account a suitable partial order, we will see that the minimal domination completions provide a decomposition of the hypergraph. We therefore conclude that any hypergraph is univocally determined by means of its minimal domination completions. In addition, we study the number of completions appearing in the decomposition of the hypergraph. This number of domination completions in a decomposition provides a new domination-related parameter. The computation of this parameter, as well as the computation of the minimal domination completions, is discussed for some special classes of hypergraphs. Proofs have been omitted due to space limitations.

\section{Recovering a graph from its dominating sets}

Here we explore whenever a graph $G$ can be univocally determined from the collection of its vertex dominating sets. We state that the graph $G$ can be uniquely determined by means of a suitable subfamily of dominating sets of vertices.

It is clear that a subset $D$ of vertices is a dominating set of the graph $G$ if and only if $D \cap N[x] \neq \emptyset$ for every vertex $x \in V(G)$, where $N[x]=\{x\} \cup\{y \in$ $V(G):\{x, y\} \in E(G)\}$ is the closed neighborhood of vertex $x$. Therefore, a subset of vertices $D$ is a dominating set of $G$ if and only if $\bigcup_{x \in D} N[x]=V(G)$.

Since any superset of a dominating set of $G$ is also a dominating set of $G$, the collection $D(G)$ of the dominating sets of a graph $G$ is a monotone increasing family of subsets of the set of vertices $V(G)$. Therefore, $D(G)$ is uniquely determined by the family $\min (D(G))$ of its inclusion-minimal elements. Let us denote by $\mathcal{D}(G)$ the family of the inclusion-minimal dominating sets of the graph $G$.

Dominating sets of a graph are closely related to independent sets. An independent set of $G$ is a set of vertices in $G$ such that no two of them are adjacent. It is clear that an independent set is also a dominating set if and only if it is an inclusion-maximal independent set. Therefore, any inclusionmaximal independent set in a graph is necessarily also an inclusion-minimal dominating set. Let us denote by $\mathcal{D}_{\text {ind }}(G)$ the collection of the inclusionmaximal independent sets of $G$. Hence, we have that $\mathcal{D}_{\text {ind }}(G) \subseteq \mathcal{D}(G) \subseteq D(G)$ 
and $\mathcal{D}_{\text {ind }}(G)=\{D \in \mathcal{D}(G): D$ is an independent set $\}$.

The following propositions state that from the collection of vertex dominating sets it is possible to recover some information concerning to the graph. Specifically, it is possible to obtain the set of edges $E(G)$ from the family $\mathcal{D}_{\text {ind }}(G)$, while the family $\mathcal{D}(G)$ allows us to recover the family $\mathcal{N}[G]$ of the inclusion-minimal closed neighborhood of the graph; that is, the family $\mathcal{N}[G]=\min \{N[x]: x \in V(G)\}$.

Proposition 2.1 Let $G$ be a graph and let $\mathcal{D}_{\text {ind }}(G)=\left\{D_{1}, \ldots, D_{t}\right\} \subseteq \mathcal{D}(G)=$ $\left\{D_{1}, \ldots, D_{s}\right\}$, where $1 \leq t \leq s$. Then,

(i) $\mathcal{N}[G]=\min \left\{\left\{a_{1}, \ldots, a_{s}\right\}: a_{i} \in D_{i}\right.$ for $\left.1 \leq i \leq s\right\}$.

(ii) $E(G)=\min \left\{\left\{a_{1}, \ldots, a_{t}\right\}: a_{i} \notin D_{i}\right.$ for $\left.1 \leq i \leq t\right\}$.

Proposition 2.2 Let $G_{1}$ and $G_{2}$ be two graphs with the same vertex set. Then,

(i) $\mathcal{D}\left(G_{1}\right)=\mathcal{D}\left(G_{2}\right)$ if and only if $\mathcal{N}\left[G_{1}\right]=\mathcal{N}\left[G_{2}\right]$.

(ii) $\mathcal{D}_{\text {ind }}\left(G_{1}\right)=\mathcal{D}_{\text {ind }}\left(G_{2}\right)$ if and only if $G_{1}=G_{2}$.

\section{Domination completions and decomposition of hyper- graphs}

Let $\Omega$ be a non-empty finite set. A hypergraph on $\Omega$ is a collection $H$ of nonempty different subsets of $\Omega$, none of which is a proper subset of another; that is, if $A, A^{\prime} \in H$ and $A \subseteq A^{\prime}$ then $A=A^{\prime}$. We denote by $\operatorname{Hyp}(\Omega)$ the collection of hypergraphs $H$ on $\Omega$ and by $\operatorname{Hyp}_{0}(\Omega)$ the collection of the hypergraphs $H$ on $\Omega$ with $\Omega=\bigcup_{A \in H} A$.

If $G$ is a graph with vertex set $V(G)=\Omega$, we consider the collection $\mathcal{D}(G)$ of the inclusion-minimal dominating sets of the graph. It is clear that $\mathcal{D}(G)$ is a hypergraph on $\Omega$. Moreover, it is straightforward to prove that the equality $\Omega=\bigcup_{A \in \mathcal{D}(G)} A$ holds. Therefore, $\mathcal{D}(G) \in \operatorname{Hyp}_{0}(\Omega) \subseteq \operatorname{Hyp}(\Omega)$.

We will say that a hypergraph $H$ on $\Omega$ is a domination hypergraph if there exists a graph $G$ with vertex set $\Omega$ such that $H=\mathcal{D}(G)$. So, if $H$ is a domination hypergraph on $\Omega$, then $H \in \operatorname{Hyp}_{0}(\Omega) \subseteq \operatorname{Hyp}(\Omega)$. Observe that there exist domination hypergraphs $H$ with more than one graph realization; that is, there exists hypergraphs $H$ such that $H=\mathcal{D}(G)=\mathcal{D}\left(G^{\prime}\right)$ with $G, G^{\prime}$ two different graphs, even more, with $G, G^{\prime}$ two non-isomorphic graphs.

Not all hypergraphs are domination hypergraphs. For instance, the hypergraph $H=\{\{1,2,3\},\{1,2,4\},\{1,3,4\},\{2,3,4\}\}$ on the finite set $\Omega=$ 
$\{1,2,3,4\}$ is not a domination hypergraph. Moreover, it is not hard to prove that $H \nsubseteq \mathcal{D}(G)$ if $G$ is a graph with vertex set $\Omega$. From this example it follows that, in general, given a hypergraph $H$ on a finite set $\Omega$ there does not exist a graph $G$ such that $H \subseteq \mathcal{D}(G)$.

A natural question that arises at this point is to determine domination completions $H^{\prime}$ of $H$; that is, domination hypergraphs $H^{\prime}$ close to $H$. The following issues are two crucial points in order to look for the domination completions of $H$. On one hand, to take into account all the dominating sets of a graph $G$ instead of considering only the inclusion-minimal dominating sets of $G$; that is, to take into account the family $D(G)$ instead of the family $\mathcal{D}(G)$. On the other hand, to consider the domination hypergraphs $H^{\prime}$ of the form $H^{\prime}=\mathcal{D}\left(G^{\prime}\right)$ for some graph $G^{\prime}$ with vertex set $V\left(G^{\prime}\right) \subseteq \Omega$ instead of those graphs with $V\left(G^{\prime}\right)=\Omega$.

In order to state our results, we must first introduce some general definitions and notations. Let $\Omega$ be a finite set. Let $H$ be a hypergraph on $\Omega$. Then we define $H^{+}$as the family whose elements are the subsets $A \subseteq \Omega$ such that there exists $A_{0} \in H$ with $A_{0} \subseteq A$. Observe that $H^{+}$is a monotone increasing family of subsets of $\Omega$ whose inclusion-minimal elements are the subsets of $H$; that is, $H=\min \left(H^{+}\right)$. Therefore, the hypergraph $H$ is uniquely determined by the monotone increasing family $H^{+}$. For instance, if $G$ is a graph then $\mathcal{D}(G)$ is a hypergraph whose associated monotone increasing family of subsets is $\mathcal{D}(G)^{+}=D(G)$, and so, $D(G)$ is uniquely determined by $\mathcal{D}(G)$.

To compare two hypergraphs $H_{1}, H_{2}$ on $\Omega$ we use their associated monotone increasing families of subsets $H_{1}^{+}, H_{2}^{+}$. It is clear that if $H_{1} \subseteq H_{2}$, then $H_{1}^{+} \subseteq$ $H_{2}^{+}$. However, the converse is not true; that is, there exist hypergraphs with $H_{1} \nsubseteq H_{2}$ and $H_{1}^{+} \subseteq H_{2}^{+}$(for instance the hypergraphs $H_{1}=\{\{1,2\},\{1,3\}\}$ and $\left.H_{2}=\{\{1,2\},\{3\}\}\right)$.

This fact leads us to consider a binary relation $\leqslant$ defined on the set of hypergraphs on $\Omega$. Namely, if $H_{1}$ and $H_{2}$ are two hypergraphs on $\Omega$, then we say that $H_{1} \leqslant H_{2}$ if and only if $H_{1}^{+} \subseteq H_{2}^{+}$. In other words, we have that $H_{1} \leqslant H_{2}$ if and only if for all $A_{1} \in H_{1}$ there exists $A_{2} \in H_{2}$ such that $A_{2} \subseteq A_{1}$. It is straightforward consequence of the definitions that the binary relation $\leqslant$ is a partial order on the set of hypergraphs on $\Omega$.

By using this partial order $\leqslant$ we define the domination completions of a hypergraph $H$ as follows. Let us denote by $\operatorname{DomHyp}_{0}(\Omega)$ the set whose elements are the domination hypergraphs on $\Omega$, and let us denote by $\operatorname{DomHyp}(\Omega)$ the set whose elements are the domination hyperpraphs $H^{\prime}$ on subsets $\Omega^{\prime}$ of $\Omega$. Let $H$ be a hypergraph on the finite set $\Omega$. A domination completion of $H$ is any domination hypergraph $H^{\prime} \in \operatorname{DomHyp}(\Omega)$ such that $H \leqslant H^{\prime}$. We 
denote by $\operatorname{Dom}(H)$ the set whose elements are the domination completions of the hypergraph $H$; that is,

$$
\operatorname{Dom}(H)=\left\{H^{\prime} \in \operatorname{DomHyp}(\Omega): H \leqslant H^{\prime}\right\} \subseteq \operatorname{Hyp}(\Omega) .
$$

The following theorem deals with the existence of domination completions of a given hypergraph.

Theorem 3.1 Let $H$ be a hypergraph on a finite set $\Omega$. Then $(\operatorname{Dom}(H), \leqslant)$ is a non-empty partially ordered set, and this poset has a unique minimal element if and only if the hypergraph $H$ is a domination hypergraph.

Remark 3.2 Given a hypergraph $H$ on a finite set $\Omega$, we can consider the set $\operatorname{Dom}_{0}(H)=\left\{H^{\prime} \in \operatorname{DomHyp}_{0}(\Omega): H \leqslant H^{\prime}\right\}$. Observe that $\operatorname{Dom}_{0}(H) \subseteq$ $\operatorname{Dom}(H)$ because $\operatorname{DomHyp}_{0}(\Omega) \subseteq \operatorname{DomHyp}(\Omega)$. It is easy to verify that $\left(\operatorname{Dom}_{0}(H), \leqslant\right)$ is a non-empty partially ordered set. However, the result in Theorem 3.1 does not work if we consider $\operatorname{Dom}_{0}(H)$ instead of $\operatorname{Dom}(H)$. Namely, there exist hypergraphs $H$ such that the poset $\left(\operatorname{Dom}_{0}(H), \leqslant\right)$ has a unique minimal element $\mathcal{D}(G)$ but $H \neq \mathcal{D}(G)$.

Let $H$ be a hypergraph. We say that a domination hypergraph $H^{\prime}$ is a minimal domination completion of $H$ if $H^{\prime}$ is a minimal element of the poset $(\operatorname{Dom}(H), \leqslant)$. Let us denote by $\operatorname{Dom}(H)$ the set whose elements are the minimal dominating completions $H^{\prime}$ of $H$; that is,

$$
\operatorname{Dom}(H)=\min (\operatorname{Dom}(H), \leqslant) .
$$

We have seen in Theorem 3.1 that if $\mathcal{D} o m(H)$ has cardinality one, then the hypergraph $H$ is a domination hypergraph. The following theorem and corollary deal with the case of cardinality greater than one, and show that we can recover uniquely the hypergraph from the elements of $\mathcal{D} o m(H)$.

Theorem 3.3 Let $H$ be a hypergraph on a finite set $\Omega$ and let $\operatorname{Dom}(H)=$ $\left\{H_{1}, \ldots, H_{r}\right\}$. Then $H=\min \left\{A_{1} \cup \cdots \cup A_{r}: A_{i} \in H_{i}\right.$ for $\left.1 \leq i \leq r\right\}$.

Corollary 3.4 Let $H_{1}$ and $H_{2}$ be two hypergraphs on a finite set $\Omega$. Then, $H_{1}=H_{2}$ if and only if $\mathcal{D o m}\left(H_{1}\right)=\operatorname{Dom}\left(H_{2}\right)$.

The previous results lead us to the following definition. Let $H$ be a hypergraph on a finite set $\Omega$. We say that a family $\left\{H_{1}, \ldots, H_{t}\right\} \subseteq \operatorname{DomHyp}(\Omega)$ of $t \geq 1$ distinct domination hypergraphs on $\Omega$ is a $t$-decomposition of the hypergraph $H$ if $H=\min \left\{A_{1} \cup \cdots \cup A_{t}: A_{i} \in H_{i}\right.$ for $\left.1 \leq i \leq t\right\}$. Let us denote $\mathfrak{D}(H)=\min \{t$ : there exists a $t$-decomposition of $H\}$. It is clear that $\mathfrak{D}(H)=1$ if and only if $H$ is a domination hypergraph. 
From Theorem 3.3 we get that the domination hypergraphs in $\operatorname{Dom}(H)$ provide a decomposition of $H$ and, therefore, if $H$ has $r$ minimal domination completions then $\mathfrak{D}(H) \leq r$.

\section{Computation of minimal domination completions}

In general, there is no description of the domination hypergraphs on a finite set $\Omega$ of size $n$, therefore the computation of the minimal domination completions of $H$ is a problem that is far from being solved. This leads us to consider some special classes of hypergraphs, namely, hypergraphs on a finite set of small size and uniform hypergraphs. A complete result has been obtained for $n \leq 4$. We give an example of minimal domination completions and decomposition of a hypergraph on $\Omega=\{1,2,3,4\}$.

Example 4.1 Let us consider the hypergraph $H=\{\{1,3\},\{2,4\}\}$ on the set $\Omega=\{1,2,3,4\}$. It can be shown that $H$ is not a domination hypergraph and has six minimal domination completions, $\operatorname{Dom}(H)=\left\{H_{1}, H_{2}, H_{3}, H_{4}, F_{1}, F_{2}\right\}$, where $H_{1}=\{\{1\},\{2,4\}\}, H_{2}=\{\{2\},\{1,3\}\}, H_{3}=\{\{3\},\{2,4\}\}, H_{4}=$ $\{\{4\},\{1,3\}\}, F_{1}=\{\{1,3\},\{1,4\},\{2,3\},\{2,4\}\}$, and $F_{2}=\{\{1,2\},\{1,3\}$, $\{2,4\},\{3,4\}\}$. For all $i \in\{1,2,3,4\}, H_{i}$ is the domination hypergraph of a path of order 3 and, for $i \in\{1,2\}, F_{i}$ is the domination hypergraph of a path of order 4. By applying Theorem 3.3 it follows that $2 \leq \mathfrak{D}(H) \leq 6$ and $\left\{H_{1}, H_{2}, H_{3}, H_{4}, F_{1}, F_{2}\right\}$ is a 6 -decomposition of $H$. However, it is easy to check that $\left\{H_{1}, H_{2}\right\}$ is a 2-decomposition of $H$, implying that $\mathfrak{D}(H)=2$.

We now point out some results concerning uniform hypergraphs. Let $\Omega=\{1, \ldots, n\}$ and let $1 \leq r \leq n$. The $r$-uniform hypergraph $U_{r, n}$ of order $n$ is the hypergraph $U_{r, n}=\{A \subseteq \Omega:|A|=r\}$. The following proposition gives a characterization of uniform hypergraphs $U_{r, n}$ that are domination hypergraphs.

Proposition 4.2 The uniform hypergraph $U_{r, n}$ is a domination hypergraph if and only if $r=1$, or $r=n$, or $r=2$ and $n$ is even. Moreover, (i) the complete graph with vertex set $\Omega$ is the unique graph $G$ such that $U_{1, n}=\mathcal{D}(G)$; (ii) the empty graph with vertex set $\Omega$ is the unique graph $G$ such that $U_{n, n}=\mathcal{D}(G)$; and (iii) there are $(2 m) ! /\left(2^{m} m !\right)$ graphs $G$ such that $U_{2,2 m}=\mathcal{D}(G)$, namely, $G$ is any graph obtained from the complete graph on $\Omega$ by deleting the edges of a perfect matching.

The study of the uniform hypergraphs will be completed with the computation of the minimal domination completions of $U_{r, n}$ either when $3 \leq r \leq n-1$ 
or when $r=2$ and $n$ is odd. Here we present the results obtained in two cases, the case $r=2$ and $n$ odd and the case $r=n-1$.

Proposition 4.3 The uniform hypergraph $U_{2,2 m+1}$ has $2 m+1$ minimal domination completions and $\mathfrak{D}\left(U_{2,2 m+1}\right)=2$.

Proposition 4.4 The minimal completions of $U_{n-1, n}$ are the hypergraphs obtained as $\mathcal{D}(G)$, where $G$ is a graph with vertex set $\{1, \ldots, n\}$ whose connected components are nontrivial stars. Moreover, $\mathfrak{D}\left(U_{3,4}\right)=3$ and $\mathfrak{D}\left(U_{n-1, n}\right) \leq n-1$ for $n \geq 5$.

\section{References}

[1] Berge, C., "Hypergraphs: Combinatorics of Finite Sets", North Holland, Amsterdam, 1989.

[2] Boros, E., V. Gurvich and I. E. Zverovich, Neighborhood hypergraphs of bipartite graphs, J. Graph Theor. 58 (2008), 69-95.

[3] Bourgeois, N., F. Della Croce, B. Escoffier and V.Th. Paschos, Fast algorithms for MIN INDEPENDENT DOMINATING SET, Discrete Appl. Math. 161 (2013), 558-572, doi:10.1016/j.dam.2012.01.003.

[4] Couturier, J-F., P. Heggernes, P. van’t Hof and D. Kratsch, Minimal dominating sets in graph classes: Combinatorial bounds and enumeration, Theor. Comput. Sci. 487 (2013), 82-94, doi:10.1016/j.tcs.2013.03.026.

[5] Fomin, F.V., F. Grandoni, A.V. Pyatkin and A.A. Stepanov, Combinatorial bounds via measure and conquer: Bounding minimal dominating sets and applications, ACM Trans. Algorithms 5 (2008), Article 9, doi:10.1145/1435375.1435384.

[6] Goddard, W., and M.A. Henning, Independent domination in graphs: A survey and recent results, Discrete Math. 313 (2013), 839-854.

[7] Haynes, T.W., S.T. Hedetniemi and P.J. Slater, "Fundamentals of Domination in Graphs", Marcel Dekker, New York, 1998.

[8] Haynes, T.W., S.T. Hedetniemi and P.J. Slater (Eds.), "Domination in Graphs: Advanced Topics", Marcel Dekker, New York, 1998.

[9] Kanté, M.M., V. Limouzy, A. Mary and L. Nourine, On the enumeration of minimal dominating sets and related notions, SIAM J. Discrete Math., 28 (2014), 1916-1929, doi:10.1137/120862612. 